\title{
Design of a Continuous Intra-Oral Distraction Device for Mandibular Bone
}

\author{
Karolina Wszelak, Mateusz Labul
}

\begin{abstract}
In this paper we present example of a specific device used for distraction osteogenesis. Distraction osteogenesis (also called callus distraction, collotasis or osteodistraction) is a surgical process for reconstructing bone deformities or lengthening bones.
\end{abstract}

Index Terms - distraction, mandibular, osteogenesis.

\section{INTRODUCTION}

The principal steps of every distraction processes are:

1. Osteotomy:

- It is a surgical operation whereby a bone is cut to lengthen, shorten or change its alignment. This procedure can be used, for example, to relieve pain in arthritis, correct hallux valgus or in distraction osteogenesis.

- In this phase, beside the cutting of the bone, the distraction device is attached to the bone.

2. Latency:

- This phase takes $4-7$ days. During this time the healing process starts, which is constitution of the distraction callus (or regenerate) across the gap to form the granular tissue.

3. Distraction:

- The main process which is the activation of the distraction device by the patient.

- In this phase the callus is changing its shape and size.

4. Consolidation:

- In this step the bone segments are in their final, fixed positions.

- The properties of regenerate are evolving.

- The consolidation takes as many days as twice the number of millimeters of the distraction.

5. Material removal:

It happens during another surgical procedure. There are three types of distraction osteogenesis, which shows Figure 1:

- unifocal- distraction consists of a single osteotomy with distraction forces applied on either side of the osteotomy,

- bifocal- distraction involves a single osteotomy separated by a transport segment (a piece of bone

Karolina Wszelak, Department of Manufacturing and Production Engineering, Faculty of Mechanical Engineering, Gdansk University of Technology, Poland.

Mateusz Labul, Faculty of Mechanical Engineering, Gdansk University of Technology, Poland. that will be distracted into the defect with new bone formation filling the defect behind the transport segment),

- trifocal- distraction that uses two osteotomies and two transport segments to fill a skeletal defect bidirectionally [1].

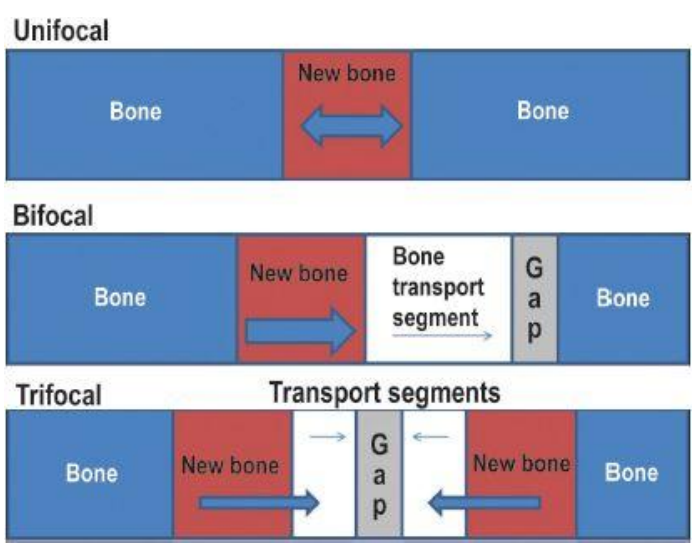

Figure 1.Types of osteodistraction [2]

In our project we are focusing on bifocal osteodistraction in mandibular bone. For such cases numerous distraction devices can be found. Unfortunately many of them are outside of the jaw, which is more painful, makes additional wounds to

patient and also affects negatively psyche of the wearer. That is why our project is so important.

II. Technical specifications

In this project we were obliged to follow some specifications. The most important criteria are continuous way of displacement and also that this device should be intraoral.

Continuity of our device will give us more or less stable force acting on our cut part of bone. This continuous load have big influence on creation of our new bone. If this requirement will be done our new modeled bone is going to be more regular. So we tried to match our device as much as possible to this criteria to obtain the best result in modeling our mandibular bone.

The second requirement about intraoral device is linked to patient. His self-confidence and good state of psychological health. Our apparatus is considered as medical device, so it has to fulfill some other requirements. The most important is biocompatibility, because it has permanent contact with human body during time of implantation. Before usage our device has to be sterilized. As material we can use alloy of titanium, because it has perfect biocompatibility and mechanical properties. After use apparatus should be utilized. 
In our case we simplified geometry of movement of our bone only to one plane.

III. Technical drafts

During our work on this project we were able to prepare four drafts for this kind of problem.

\section{A. First draft}

In the first draft that we thought of we wanted to create a device with pneumatic actuator. The direction of the motion would be controlled by two rails. The whole device would be fixed to the bone by standard foot-plates. Unfortunately is not possible to use any kind of automatic mechanism in mouth. That is why this draft was rejected at the very beginning of our work.

\section{B. Second draft}

The second draft is based on a curved tube with cut pocket at the bottom. The motion would be controlled by the mechanism consisted of bendable gear rack (connected with moving part) and gear wheel. The gear rack would be pulled by the rotating gearwheel thus making the transport segment moving. The biggest problem of this concept was its mechanism. To make a real continuous motion, patient would have to rotate the gearwheel all the time. It is of course impossible so this idea was also not good enough.

\section{Third draft}

In the third draft we focused on springs. They were perfect for making a continuous motion of the device. Similar to the second draft, in here we have a tube in which inside would be spring. The spring would push the tiny cylinder that would be connected directly to the transport segment. There were a couple of problems with this design. First of all it was impossible to find one, perfect spring for such purpose. That is why we wanted to let patient add or swap additional springs from the front of the device after a couple of days. As it turned out in our case the transport segment has to be transported from the back to the front which makes it impossible for the patient to add/swap the springs. Another problem was the continuous and safe aspect. In this case it was difficult to predict how the spring will behave and if it is going to push the cylinder with constant force. To fix it we were thinking about some kind of stopping device that would be placed on the rail parallel to the tube.

\section{Fourth draft}

The fourth draft is the combination of the second and third concept. In here the role of the controlling and stopping device have the short tube that is moving between two parallel rails. In the tube there is a short cylinder that is connected to the transport segment (similar to the third draft). The spring is also inside the tube to push the cylinder giving the motion to the transport segment. However the whole tube is moved thanks to the bendable gear rack. The gear rack is connected to the tube and is pulled by the wheel with one tooth to make

sure that patient is not going to move the device too much (which is of course similar to our second concept). After the gear rack is going be pulled too much, patient will be able to just cut out the excess.

This is the concept that we chose to create CAD model. It makes simple and continuous motion of the transport segment and it is easy and safe to use by the patient.

\section{Technical solutions and CAD model}

As we stated in the previous chapter, as a final draft we chose our fourth concept. In our opinion this one was best suitable for our task. It provides sure way of transporting the bone segment and is completely intra-oral. We were able to create the model of our device in Catia software. In the Figure 2 it can be seen how this device would look like on the mandibular bone.

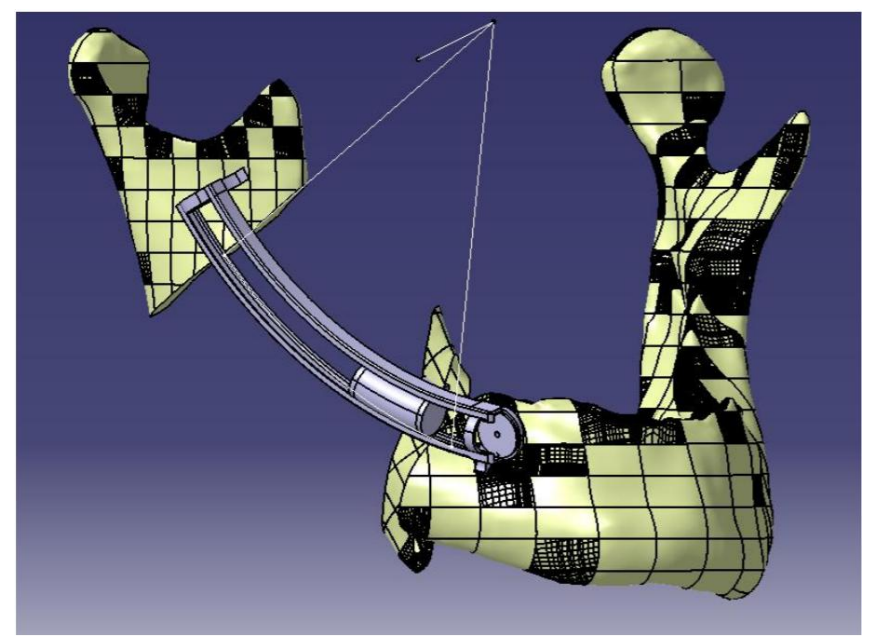

Figure 2. Model of the distraction device

As a material for this device we chose titanium. Thanks to its biocompatibility, mechanical properties and the fact that not many people are allergic for it, makes it a perfect choice for our purpose.

The crucial part of our device is the spring. We had to make sure, that it is going to:

- be biocompatible,

- be able to push the bone with the force of $20 \mathrm{~N}$,

- will not be too big for the device.

Fortunately we were able to find it- compressing spring C.056.100.0111.I. It is made from stainless steel, it is able to push with the exact force of $20 \mathrm{~N}$ and its outside diameter is equal to 5,6 mm [4]. Additionally, when the spring is going to be maximum pressed, its length is going to be $7 \mathrm{~mm}$. Without compression it will have length equal to $8 \mathrm{~mm}$ which is the perfect amount for us, because it gives us $1 \mathrm{~mm}$ of elongation. This spring is going to be placed inside a small tube. The whole idea behind the spring and the distraction can be easily explained after looking at Figure 3.

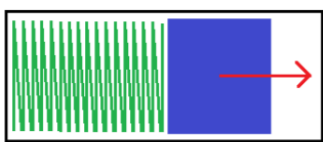

a)

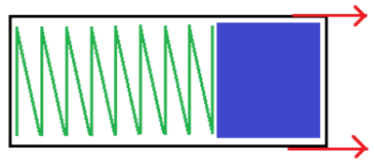

b)

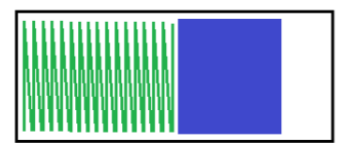

c)

Figure 3. How distracting mechanism works: a) The spring is compressed; it pushes the cylinder (that is connected with transported bone). b) Cylinder cannot go any further; patient is pulling the tube further. c) Cylinder will be pushed again by 
the spring.

V. conclusion

Working on this project helped us realize how complicated is the process of creating a medical device. There are many aspects that engineer has to remember while designing such a device, like biocompatibility or biodegradability (not in our case). Moreover, devices usually has to be made for specific patient, so there is always a problem of perfect dimensions for the person.

Our two main requirements in this projects were continuous way of distraction and designing the device to be intraoral. We succeeded to fulfill both of them. The system based on spring and gear rack makes it possible for our device to work continuously. Additionally the small dimensions of the device and possibility to activate it through open mouth makes it completely intraoral.

Our device is able to work in case of bone loss length of 50 $\mathrm{mm}$ and the every-day distraction equals $1 \mathrm{~mm}$. Thanks to choosing the biocompatible stainless steel we are sure it can be sterilized and utilized. This device is also safe for a patient, because it does not have sharp edges and the gear mechanism prevents the possibility of too big or too small distraction. Our product is also easy to use- patient needs only to spin one gear wheel once a day. Thanks to the material chosen and small dimensions of the device we are sure it will not weight more than $50 \mathrm{~g}$. Finally it will be also easy to install by the surgeon thanks to unique shapes of each parts.

This project was not so easy because there are not this kind of devices on the market, so we had to find the solution without any help. However we think our device managed to fulfill basic requirements and would be a big help for patients with mandibular bone defects.

\section{REFERENCES}

[1] Cai M1, Lu X, Shen G, Wang X, Cheng AH.; Customized bifocal and trifocal transport distraction osteogenesis device for extensive mandibular reconstruction.

[2] Edward J. Caterson, Joseph G. McCarthy,; Principles and Practice of Mandibular Distraction Osteogenesis

[3] http://www.medicalexpo.com/prod/ningbo-cibei-medical-treatment-ap pliance/product-69525-460024.html

[4] https://www.vanel.com/compression-adv-search.php?lang=polish\&ses sionid $=19350135195566 \mathrm{a} 7 \mathrm{~d} 26445 \mathrm{~cd} 385840761301284193640731 \# \mathrm{re}$ sults

[5] Uriel Zapata, Mohammed E. Elsalanty, Paul C. Dechow, Lynne A. Opperman,;Biomechanical Configurations of Mandibular Transport Distraction Osteogenesis Devices 